\title{
LINE-PROFILE VARIABILITY OF EZ CMA
}

\author{
L. GEORGIEV and M.M. IVANOV \\ Department of Astronomy, University of Sofia, 5 James Bourchier st., BG-1126 Sofia, \\ Bulgaria
}

\begin{abstract}
CCD spectra for the unusual Wolf-Rayet star EZ CMa (WR6) are presented. The analysis of the profile changes of HeII lines shows a correlation between the gravity center of the line and the skewness (Robert et al. 1992). The fourth moment (kurtosis) changes with phase and looks epoch independent. These observable effects could be treated as manifestations of wind variability rather than consequence of orbital motion.
\end{abstract}

Key words: stars: Wolf-Rayet - stars individual: WR6

We present new CCD high-resolution spectra for EZ CMa, obtained at the NAO "Rozhen". This unusual WR star is well known for its unique spectral, photometric and polarimetric behaviour. The proposed binary WR+c (c = compact companion) model (Firmani et al. 1980) is questioned because of: (1) lack of strong X-ray emission (Pollock 1989); (2) absence of phase variations due to the Hatchett-McCray effect; (3) epoch-dependence of light-, polarization- and radial-velocity curves. Alternatives for the binary hypothesis are: (a) a rotating, expanding wind geometry around a single star (Schulte-Ladbeck et al. 1990); (b) a rotating disk with central filaments (Underhill and Yang 1991); (c) bipolar outflow (Matthews et al. 1992); (d) non-radial pulsations of a single star (Gosset and Vreux 1987).

The purpose of this paper is to discuss the variability of He II spectral lines in the light of single and binary star hypotheses.

Our data show short time-scale variability of the fine detailes superposed on the He II $5412 \AA$ line wings, and global, phase dependent $(P=3.766 \mathrm{~d})$ profile changes interpreted as radial velocity variations in previous papers. From the observed correlation between the center of gravity and the skewness of the line it is obvious that it is easy to confuse an actual shift of the line with a change of its symmetry.

To avoide the problem mentioned above, we choose to study the fourth moment (kurtosis) of the line. The maximum of the kurtosis corresponds to a 'flat-top profile', whereas the minimum - to a 'sharp-top profile'. This is independent of any shifts of the line, but reflects the changes of the optical depth and/or geometry of a line-forming region. In Fig. 1 (left) the fourth moment is plotted vs. time. The presence of long-time scale variations is obvious. After subtraction of this trend, the noise in the 'light-curve' of the kurtosis is hardly reduced (Fig.2 right). Therefore, if we look at the 'flatness' of the line there are no phase jumps, but only underlying long-time scale $(P>>100 \mathrm{~d})$ amplitude variations. As we mentioned above, the fourth moment is related to the physics of the emitting region, and its variations 

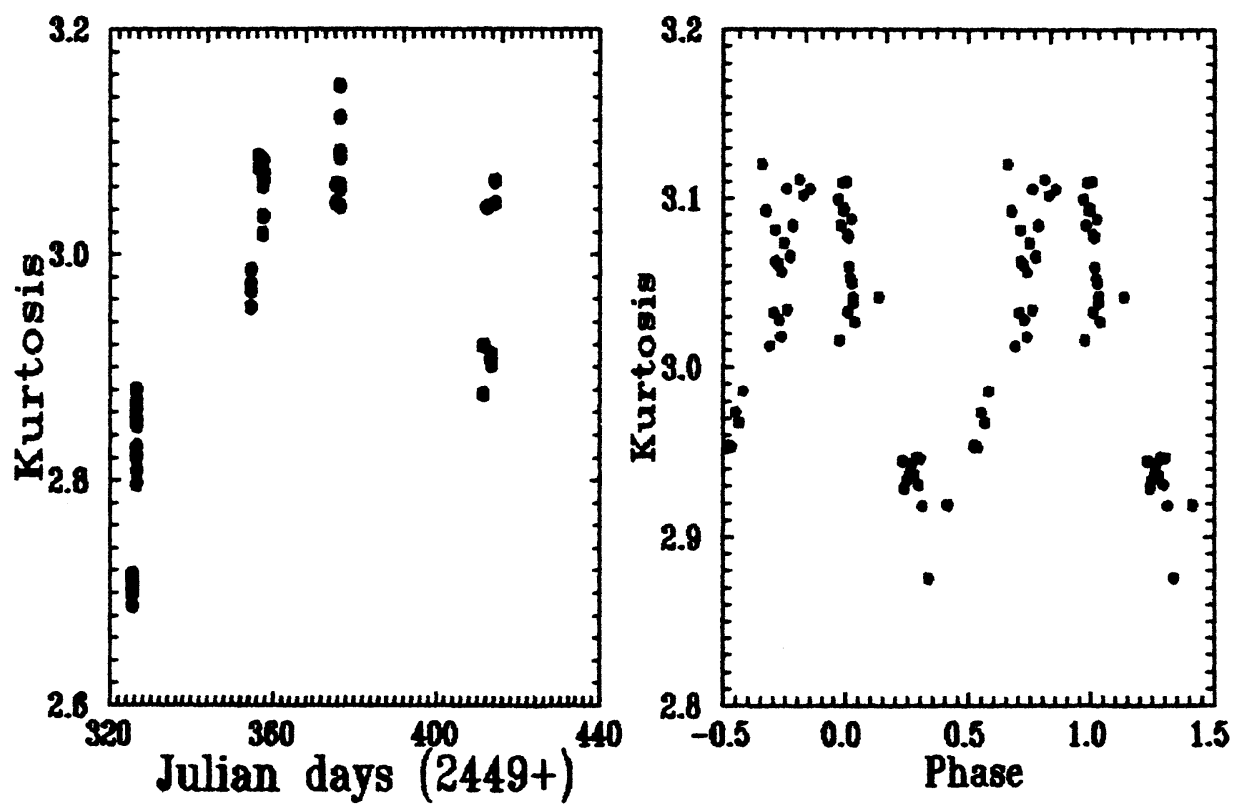

Fig. 1. The variations of the kurtosis of HeII $5412 \AA$ vs. time (Julian days, left) and phase $(P=3.766 \mathrm{~d})$

could be connected with rotational modulation of the physical phenomenon, rather than with binary modulation. Therefore, we conclude that the profile variations could be related to intrisic instabilities in a single star wind rather than to a binary star.

\section{Acknowledgements}

This research was supported by the Bulgarian National Scientific Foundation grant under contracts No. F-67/1992 and 3017/1993.

\section{References}

Firmani, C., Koenigsberger, G., Bisiacchi, G.F., Moffat, A.F.J., Isserstedt, J. 1980, ApJ 239, 607

Gosset, E., Vreux, J.-M. 1987, $A \& A$ 178, 153

Matthews, J.M., St-Louis, N., Moffat, A.F.J., Drissen., L., Koenigsberger, G., Cardona, O., Niemela, V.S. 1992, in: L. Drissen, C. Leitherer \& A. Nota (eds.), Non-isotropic and Variable Outflows from Stars, ASP Conf. Series 22, p. 130.

Pollock, A.M.T. 1989, ApJ 347, 409

Robert, C., Moffat, A.F.J., Drissen, L., Lamontagne, R.,Seggewiss, W., Niemela, V.S., Cerruti, M.A., Barrett, P., Bailey, J., Garcia, J.,Tapia, S. 1992, ApJ 397, 277

Schulte-Ladbeck, R.E., Nordsieck, K.H., Nook, M.A., Magalhães, A.M., Taylor, M., Bjorkman, K.S., Anderson, C.M. 1990, ApJ (Letters) 365, L19.

Underhill, A.B., Yang, S. 1991, ApJ 368, 588 